\title{
The Effect of Moderate- and High-Fat Meals on the Bioavailability of Dolutegravir/Rilpivirine Fixed-Dose Combination Tablet
}

This article was published in the following Dove Press journal: Clinical Pharmacology: Advances and Applications

\author{
Rashmi Mehta' \\ Joseph Piscitelli ${ }^{2}$ \\ Allen Wolstenholme ${ }^{3}$ \\ Caifeng $\mathrm{Fu}^{4}$ \\ Herta Crauwels ${ }^{5}$ \\ Brian Wynne ${ }^{6}$ \\ Kimberly Adkison (1D ${ }^{7}$ \\ 'GlaxoSmithKline, Research Triangle \\ Park, NC, USA; ${ }^{2}$ UNC Eshelman School \\ of Pharmacy, Chapel Hill, NC, USA; \\ ${ }^{3}$ GlaxoSmithKline, Collegeville, PA, USA; \\ ${ }^{4}$ PAREXEL International, Durham, NC, \\ USA; ${ }^{5}$ Janssen Pharmaceutical Companies, \\ Beerse, Belgium; ${ }^{6} \mathrm{Vii}$ Healthcare, \\ Collegeville, PA, USA; ${ }^{7}$ ViiV Healthcare, \\ Research Triangle Park, NC, USA
}

\begin{abstract}
Dolutegravir $50 \mathrm{mg}$ (DTG) and rilpivirine $25 \mathrm{mg}$ (RPV) are a newly approved 2-drug regimen for the treatment of HIV in virally suppressed patients. A 2-part study evaluated the relative bioavailability and food effect of five experimental fixed-dose combination (FDC) tablet formulations of DTG/RPV. When given with a moderate- or high-fat meal, the absorption of both DTG and RPV was increased, resulting in higher exposures. As per product labelling, DTG/RPV FDC should be taken with a meal.
\end{abstract}

Keywords: food effect, pharmacokinetics, dolutegravir, rilpivirine

Juluca $^{\circledR}$, (ViiV Healthcare) the 2-drug regimen (2-DR) of dolutegravir $50 \mathrm{mg}$ (DTG) and rilpivirine $25 \mathrm{mg}$ (RPV), administered once daily with a meal has been approved in many countries for the treatment of HIV-1 infection in virologically suppressed patients. The approvals were based on the results of two Phase 3 trials (SWORD 1 and 2) which demonstrated non-inferior efficacy and safety of DTG + RPV once daily with a meal when compared to current 3-drug antiretroviral therapy, ${ }^{1}$ and a pivotal bioequivalence (BE) study in fed condition which demonstrated that a fixed-dose combination tablet (DTG/RPV) was bioequivalent to the single entity tablets. ${ }^{2}$

During the course of drug development, a relative bioavailability (BA) study was conducted to evaluate the pharmacokinetics of multiple experimental fixeddose combination (FDC) formulations in both the fasted and fed states in order to select the best FDC tablet formulation and to estimate food effect. ${ }^{3}$ This communication will report the effect of food (moderate- and high-fat/calorie meals) on the pharmacokinetics of one of the DTG/RPV fixed-dose combination tablets: Formulation AM. Formulation AM was compositionally identical to the formulation evaluated in the pivotal BE study and eventually marketed product (DTG/RPV FDC) Juluca ${ }^{\circledR}$, except had a different color film coat and no debossing. The relative bioavailability and food effect comparisons for the other formulations were previously presented ${ }^{3}$ and will not be repeated here.

Study 201674 (NCT02373930) was a 2-part single-dose study to evaluate the relative bioavailability and food effect of five experimental FDC tablet formulations of DTG/RPV. Part 1 was a randomized, open-label, 3-way crossover, incomplete block, Youden square design in 24 healthy adult subjects with a 10-day between-treatment washout. All doses were administered $30 \mathrm{~min}$ after consumption of a high-fat meal (approximately 900 total calories: $17 \%$ protein, $28 \%$ carbohydrates, and $56 \%$ fat). ${ }^{4}$ Part
Correspondence: Kimberly Adkison ViiV Healthcare, 5 Moore Drive, Research Triangle Park, NC 27709, USA

$\mathrm{Tel}+1919483-5867$

Email kim.k.adkison@viivhealthcare.com 
Table I Effect of Moderate- or High-Fat Meals on the Pharmacokinetics of a Dolutegravir/Rilpivirine Fixed-Dose Combination Tablet (Formulation AM) in Healthy Subjects

\begin{tabular}{|c|c|c|c|c|}
\hline \multirow[t]{2}{*}{ Drug } & \multirow[t]{2}{*}{ PK Parameter } & \multicolumn{2}{|c|}{ Geometric Mean (CV\%) } & \multirow{2}{*}{$\begin{array}{l}\text { Ratio of Geometric Least Squares Means ( } 90 \% \\
\text { Confidence Interval) Fed vs Fasted }\end{array}$} \\
\hline & & Fed $N=12$ & Fasted $\mathbf{N}=12$ & \\
\hline \multicolumn{5}{|c|}{ Moderate-Fat Meal ${ }^{\mathrm{a}}$ (Part 2) vs Fasted (Part 2) Crossover Comparison } \\
\hline Dolutegravir & $\begin{array}{l}\operatorname{A\cup C}(0-\infty)(\mu g . h / m L) \\
C \max (\mu g / m L) \\
\operatorname{Tmax}(h)^{c}\end{array}$ & $\begin{array}{l}64.62(20.7) \\
3.40(21.2) \\
3.51(1.0,5.0)\end{array}$ & $\begin{array}{l}34.64(57.1) \\
1.94(63.2) \\
2.25(1.0,5.0)\end{array}$ & $\begin{array}{l}\mathrm{I} .865(1.542,2.257) \\
\mathrm{I} .749(1.403,2.18 \mathrm{I}) \\
\text { N/A }\end{array}$ \\
\hline Rilpivirine & $\begin{array}{l}\operatorname{AUC}(0-\infty)(\mathrm{ng} \cdot \mathrm{h} / \mathrm{mL}) \\
\mathrm{Cmax}(\mathrm{ng} / \mathrm{mL}) \\
\operatorname{Tmax}(\mathrm{h})^{c}\end{array}$ & $\begin{array}{l}3508(37.8)^{\mathrm{d}} \\
95.08(35.9) \\
5.00(2.0,5.0)\end{array}$ & $\begin{array}{l}2236(67.6)^{d} \\
50.29(92.2) \\
4.01(2.0,5.0)\end{array}$ & $\begin{array}{l}I .569(1.244,1.980)^{d} \\
I .891(1.339,2.669) \\
\text { N/A }\end{array}$ \\
\hline \multicolumn{5}{|c|}{ High-Fat Meal ${ }^{\mathrm{b}}$ (Part I) vs Fasted (Part 2) Parallel Cohort Comparison } \\
\hline Dolutegravir & $\begin{array}{l}\operatorname{A\cup C}(0-\infty)(\mu g \cdot h / m L) \\
C \max (\mu g / m L) \\
\operatorname{Tmax}(h)^{c}\end{array}$ & $\begin{array}{l}60.75(25.6) \\
3.36(18.6) \\
4.00(2.0,6.0)\end{array}$ & $\begin{array}{l}34.64(57.1) \\
1.94(63.2) \\
2.25(1.0,5.0)\end{array}$ & $\begin{array}{l}\text { I.873 (I.533, 2.289) } \\
\text { I.7I8 (I.4II, 2.092) } \\
\text { N/A }\end{array}$ \\
\hline Rilpivirine & $\begin{array}{l}\operatorname{AUC}(0-\infty)(n g . h / m L) \\
C \max (n g / m L) \\
\operatorname{Tmax}(h)^{c}\end{array}$ & $\begin{array}{l}3743(34.1)^{d} \\
107.9(45.7) \\
4.00(2.5,5.0)\end{array}$ & $\begin{array}{l}2236(67.6)^{d} \\
50.29(92.2) \\
4.01(2.0,5.0)\end{array}$ & $\begin{array}{l}1.716(1.360,2.164)^{\mathrm{d}, \mathrm{e}} \\
2.168(1.619,2.902) \\
\text { N/A }\end{array}$ \\
\hline
\end{tabular}

Notes: aModerate-fat breakfast contained $~ 625$ total calories: 125 calories from protein, 300 calories from carbohydrate, and 200 calories from fat. ${ }^{b}$ High-fat breakfast contained $\sim 900$ total calories: 150 calories from protein, 250 calories from carbohydrate, and 500 calories from fat. ${ }^{\mathrm{C}}$ Tmax presented as median (range); Tmax was not formally compared (N/A). ${ }^{d}$ Approximately $20 \%$ of PK profiles across the study had $A U C(0-\infty)$ with \% extrapolated $>20 \%$. ${ }^{e}(n=I I)$; I subject (fed) was excluded from the statistical analysis of $\mathrm{AUC}(0-\infty)$ because $\%$ extrapolated $>40 \%$ and lambda $\mathrm{z}$ time duration $<2 \times$ calculated $\mathrm{t}^{1} / 2$.

1 evaluated four different formulations (AS, AM, AQ, AK) and a reference which was 1 tablet each of DTG $\left(\right.$ Tivicay $\left.{ }^{\circledR}\right)$ and RPV (Edurant ${ }^{\circledR}$ ) taken concurrently. Part 2 was a randomized, open-label, 3-way crossover with a 10-day between-treatment washout in 3 distinct cohorts each with 12 subjects. Two experimental tablets from Part 1 (AK and AM) and one previously untested experimental tablet (AU) were selected for evaluation in Part 2. All doses were administered in the fasted state or 30 min after a moderate-fat meal (approximately 625 total calories: 20\% protein, 48\% carbohydrates, and $32 \%$ fat) in Part 2.

During both Parts 1 and 2, serial plasma PK samples were taken from 0 hours (predose) up to 168 hours after dosing and a variety of safety assessments were conducted. DTG and RPV plasma concentrations were measured by validated LC/ MS/MS assays ${ }^{5,6}$ and PK parameters were estimated by
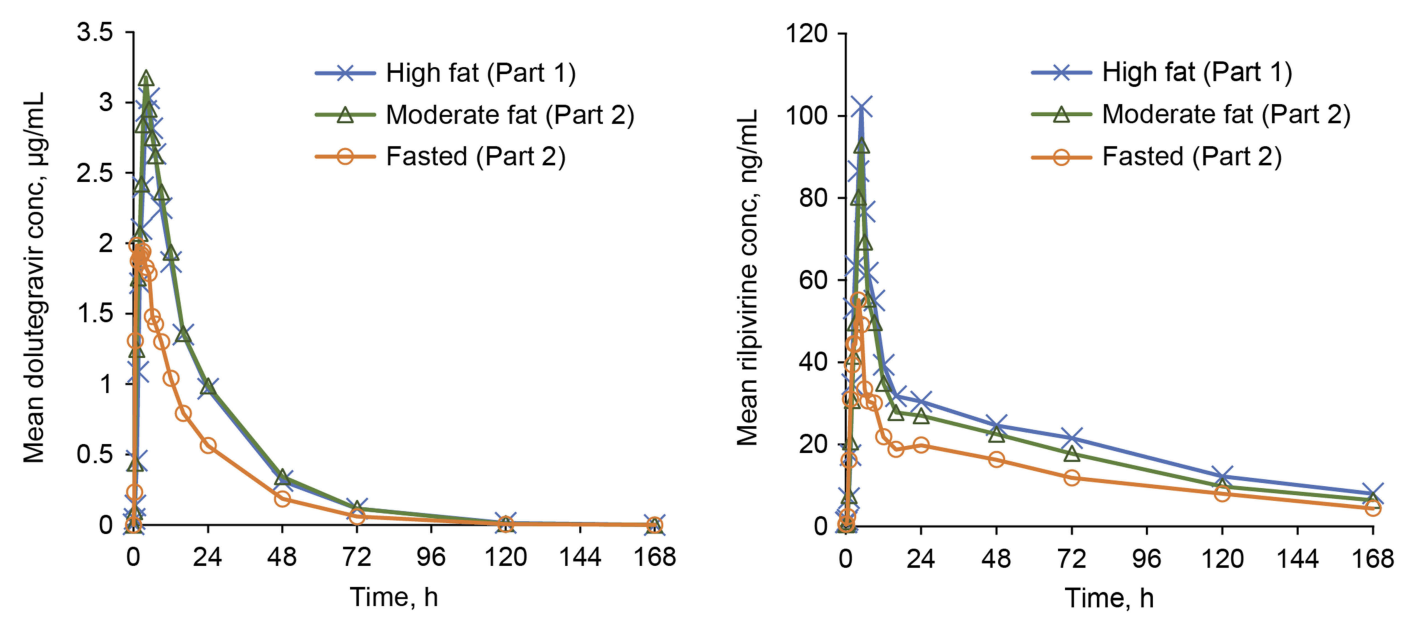

Figure I Mean plasma concentration-time profile of dolutegravir (left) and rilpivirine (right) following a single oral dose of dolutegravir/rilpivirine fixed-dose combination tablet (Formulation AM) in healthy subjects. 
noncompartmental methods using Phoenix WinNonlin (Certara, Princeton, NJ, USA). For each primary PK parameter evaluated $[\mathrm{AUC}(0-\infty)$ and $\mathrm{Cmax}$ ], the point estimates and corresponding $90 \% \mathrm{CI}$ were constructed for the ratio of the geometric least squares (GLS) means to compare the DTG and RPV PK parameters in the fed vs fasted state. The effect of a moderate-fat meal was derived from the PK results for Formulation AM from Part 2 (within-subject comparison between fasted and moderate-fat fed) and the effect of a high-fat meal on the systemic exposure of DTG and RPV was derived from cross-cohort comparisons of Formulation AM PK between Part 1 (high fat) and Part 2 (fasted).

This study was conducted in accordance with ICH GCP and the ethical principles outlined in the Declaration of Helsinki, 2013. The protocol and informed consent were approved by the Midlands Independent Review Board (Overland Park, KS, USA). Written informed consent was obtained from each subject prior to the performance of any study-specific procedures.

DTG and RPV exposures were higher when the DTG/RPV FDC was administered after consumption of a moderate- or high-fat meal than when administered in the fasted state (Table 1; Figure 1). Co-administration of DTG/RPV FDC with a moderate-fat meal increased DTG AUC $(0-\infty)$ and Cmax by $87 \%$ and $75 \%$, respectively, and RPV AUC $(0-\infty)$ and Cmax by $57 \%$ and $89 \%$, respectively. Co-administration of the DTG/RPV FDC with a high-fat meal increased DTG AUC and Cmax by $87 \%$ and $72 \%$, respectively, and RPV AUC and Cmax by $72 \%$ and $117 \%$, respectively. The study was not designed to directly compare different meal types and any apparent differences may be attributed to methodology used in comparisons (within-subject crossover for moderate fat versus cross-cohort for high fat).

In both parts of the study, drug-related adverse events (AEs) were mostly mild (abdominal pain, diarrhea, headache). Only three AEs were reported as moderate intensity (tooth abscess, headache) and no serious AEs were reported during the study. No clinically relevant findings in vital signs, ECGs, or clinical laboratory tests were reported. There were two non-serious AEs, both considered not related to study drug, leading to withdrawal from study, which included the above tooth abscess and ALT increase.

In conclusion, data collected in this relative bioavailability and food effect study allowed us to characterize the effect of moderate- and high-fat meals on the PK of the DTG/RPV FDC (Formulation AM). As expected, when the DTG/RPV fixed-dose combination tablet was taken with a meal, the absorption of both dolutegravir and rilpivirine was increased, resulting in higher DTG and RPV exposures. The magnitude of food effect was consistent with what was previously observed for the DTG and RPV single entity products ${ }^{7,8}$ and with other DTG- or RPV-containing fixed-dose combination products. ${ }^{9,10}$ While DTG is approved to be taken with or without food, taking DTG/RPV FDC in fasted condition or with only a protein-rich nutritional drink may result in decreased plasma concentrations of rilpivirine, which could potentially reduce the therapeutic effect of DTG/RPV FDC. ${ }^{7}$ DTG/RPV FDC, similar to rilpivirine-containing medicinal products, must be taken with a meal to obtain optimal absorption of rilpivirine.

\section{Disclosure}

Rashmi Mehta and Allen Wolstenholme are employees of GlaxoSmithKline. Caifeng Fu is an employee of PAREXEL International. Herta Crauwels is a full-time employee of Janssen Pharmaceutical Companies, which is owned by Johnson and Johnson, and stockholder of Johnson and Johnson, market authorisation holder of rilpivirine. Brian Wynne and Kimberly Adkison are employees of ViiV Healthcare. The authors report no other conflicts of interest in this work.

\section{References}

1. Llibre JM, Hung C-C, Brinson C, et al. Efficacy, safety, and tolerability of dolutegravir-rilpivirine for the maintenance of virological suppression in adults with HIV-1: phase 3, randomised, non-inferiority SWORD-1 and SWORD-2 studies. Lancet. 2018;391(10123):839-849. doi:10.1016/ S0140-6736(17)33095-7

2. Mehta R, Wolstenholme A, Di Lullo KD, et al. Bioequivalence of a fixed-dose combination tablet of the complete two-drug regimen of dolutegravir and rilpivirine for treatment of HIV-1 infection. Antimicrob Agents Chemother. 2018;62(9). doi:10.1128/aac.0074818

3. Adkison KK, Wolstenholme A, Choukour M, et al. Relative bioavailability and food effect of fixed-dose combination tablets of dolutegravir and rilpivirine in healthy subjects. 17th International Workshop on Clinical Pharmacology of HIV \& Hepatitis Therapy; June 8-10, 2016; Washington, DC.

4. U.S. Department of Health and Human Services Food and Drug Administration. Center for Drug Evaluation and Research. Guidance for Industry: Food-Effect Bioavailability and Fed Bioequivalence Studies; 2002.

5. Song IH, Borland J, Savina PM, et al. Pharmacokinetics of single-dose dolutegravir in HIV-seronegative subjects with moderate hepatic impairment compared to healthy matched controls. Clin Pharmacol Drug Dev. 2013;2(4):342-348. doi:10.1002/cpdd.55

6. Ford SL, Gould E, Chen S, et al. Lack of pharmacokinetic interaction between rilpivirine and integrase inhibitors dolutegravir and GSK1265744. Antimicrob Agents Chemother. 2013;57(11):5472-5477. doi:10.1128/ aac.01235-13

7. Crauwels HM, Heeswijk RPV, Buelens A, et al. Impact of food and different meal types on the pharmacokinetics of rilpivirine. $J$ Clin Pharmacol. 2013;53(8):834-840. doi:10.1002/jcph.107 
8. Song I, Borland J, Chen S, et al. Effect of food on the pharmacokinetics of the integrase inhibitor dolutegravir. Antimicrob Agents Chemother. 2012;56(3):1627-1629. doi:10.1128/AAC.05739-11

9. Weller S, Chen S, Borland J, et al. Bioequivalence of a dolutegravir, abacavir, and lamivudine fixed-dose combination tablet and the effect of food. J Acquir Immune Defic Syndr. 2014;66(4):393-398. doi:10. 1097/qai.0000000000000193
10. Ramanathan S, Custodio J, Yin X, et al. Effect of food on the pharmacokinetics of emtricitabine/rilpivirine/tenofovir disoproxil fumarate single-tablet regimen. $J$ Int AIDS Soc. 2012;15(Supp14):1. doi:10.7448/ias.15.6.18333

\section{Publish your work in this journal}

Clinical Pharmacology: Advances and Applications is an international, peer-reviewed, open access journal publishing original research, reports, reviews and commentaries on all areas of drug experience in humans. The manuscript management system is completely online and

includes a very quick and fair peer-review system, which is all easy to use. Visit http://www.dovepress.com/testimonials.php to read real quotes from published authors. 